\title{
Exact Coefficients of the Limit Cycle in Van der Pol's Equation*
}

\author{
André Deprit** and Dieter S. Schmidt \\ Department of Mathematics, University of Cincinnati, Ohio 45221 \\ March 30, 1979
}

\begin{abstract}
A program generator to manipulate automatically Poisson series over the field of rational numbers is applied to develop the limit cycle of Van der Pol's equation in the powers of the small parameter. The results indicate that the recurrence relations in what Melvin calls the algorithm of the shifted phase are stable.
\end{abstract}

Key words: Algebra by computer; differential equations; mathematical software; non-linear oscillations.

In a recent note $[1]^{1}$, Melvin presented a new set of rules to expand the limit cycle of Van der Pol's equation

$$
\ddot{y}+y=\epsilon \dot{y}\left(1-y^{2}\right)
$$

in power series of the small parameter $\epsilon$. He carried out the calculations in single precision floating point arithmetic; he suggested that they be repeated in exact form, i.e. that the coefficients in the series be produced as quotients of relatively prime integers. To achieve this result we applied to this problem a program generator of our own, called MAO, to manipulate rational Poisson series by computer. In the present note we show how this processor enables us to simplify drastically Melvin's algorithm; then we present the actual expansions for the limit cycle and its frequency to $\epsilon^{8}$.

Melvin's rules are expressed in terms of a new independent variable

$$
\tau=\omega t
$$

by means of which the original equation is transformed into

$$
\omega^{2} y^{\prime \prime}+y=\epsilon \omega y^{\prime}\left(1-y^{2}\right) .
$$

The cycle and its frequency are expanded in formal power series

$$
\begin{aligned}
& y=\sum_{n \geq 0} \frac{1}{n !} y_{n}(\tau) \epsilon^{n}, \\
& \omega=\sum_{n \geq 0} \frac{1}{n !} \omega_{n} \epsilon^{n}
\end{aligned}
$$

of the small parameter $\epsilon$. Melvin showed that the following parity rules are consistent:

(i) For $n$ even, the term $y_{n}$ is an even function of $\tau$;

(ii) For $n$ odd, the coefficient $\omega_{n}$ is 0 and the term $y_{n}$ is an odd function of $\tau$.

The terms $y_{n}$ and the coefficients $\omega_{n}$ are to be determined recursively, starting with

$$
y_{0}=2 \cos \tau \text { and } \omega_{0}=1 .
$$

\footnotetext{
* An invited paper.

** Present address: Center for Applied Mathematics, National Bureau of Standards, Washington, D.C. 20234.

${ }^{1}$ Figures in brackets indicate literature references at the end of this paper.
} 


$$
F=\epsilon \omega y^{\prime}\left(1-y^{2}\right)-\omega^{2} y^{\prime \prime}
$$

be expanded as the power series

$$
F=\sum_{n \geq 0} \frac{1}{n !} \epsilon^{n} F_{n}
$$

As we introduce the intermediate quantities

$$
u=y^{2}, \quad v=\omega y^{\prime}, \quad w=\omega^{2},
$$

we observe that the coefficients in the power series

$$
u=\sum_{n \geq 0} \frac{1}{n !} u_{n} \epsilon^{n}, \quad v=\sum_{n \geq 0} \frac{1}{n !} v_{n} \epsilon^{n}, \quad w=\sum_{n \geq 0} \frac{1}{n !} w_{n} \epsilon^{n}
$$

are given by the expressions

$$
\begin{gathered}
u_{n}=\sum_{0 \leq m \leq n}\left(\begin{array}{c}
n \\
m
\end{array}\right) y_{m} y_{n-m}, \quad v_{n}=\sum_{0 \leq m \leq n}\left(\begin{array}{c}
n \\
m
\end{array}\right) \omega_{m} y_{n-m}^{\prime} \\
w_{n}=\sum_{0 \leq m \leq n}\left(\begin{array}{c}
n \\
m
\end{array}\right) \omega_{m} \omega_{n-m},
\end{gathered}
$$

where $\left(\begin{array}{c}n \\ m\end{array}\right)$ stands for the binomial coefficient $n ! / m !(n-m) !$. Therefore

$$
F_{n}=n\left[v_{n-1}-\sum_{0 \leq m \leq n-1}\left(\begin{array}{c}
n-1 \\
m
\end{array}\right) v_{m} u_{n-1-m}\right]-\sum_{0 \leq m \leq n}\left(\begin{array}{c}
n \\
m
\end{array}\right) w_{m} y_{n-m}^{\prime \prime} .
$$

We denote by $F_{n}^{*}$ the value which $F_{n}$ takes when we set $y_{n}=0$. In view of the parity rules, $F_{n}$ is an even or odd function of $\tau$ respectively when $n$ is even or odd. Now it turns out that the term $y_{n}$ in the limit cycle is a solution of the non-homogeneous linear equation

$$
y_{n}^{\prime \prime}+y_{n}=F_{n}^{*}
$$

Hence

$$
y_{n}=y_{n}^{\circ}+y_{n}^{*}
$$

with the homogeneous general solution

$$
y_{n}^{\circ}=c_{n} \cos \tau+s_{n} \sin \tau
$$

and $y_{n}^{*}$ a particular solution of eq (3). In application of the parity rules, we set $s_{n}=0$ when $n$ is even and $c_{n}$ $=0$ when $n$ is odd. For the particular solution $y_{n}^{*}$ to be periodic in $\tau$, the right hand member $F_{n}^{*}$ must not contain terms in $\sin \tau$ and $\cos \tau$. These critical terms will be removed from $F_{n}^{*}$ by following the periodicity rules:

(i) When $n$ is even, $F_{n}^{*}$ contains a term in $\cos \tau$ whose coefficient is of the form $a-b \omega_{n}$. Therefore, we continue determining the frequency in the limit cycle by setting $\omega_{n}=a / b$. The coefficient $c_{n}$ in the term $y_{n}^{\circ}$ will be determined at the next order.

(ii) When $n$ is odd, $F_{n}^{*}$ contains a term in $\sin \tau$ whose coefficient is of the form $a-b c_{n-1}$. The determination of $y_{n-1}$ is completed by setting $c_{n-1}=a / b$. We also set $s_{n}=0$ which implies that $y_{n}^{\circ}$ $=0$ in all components of the limit cycle at odd orders. 
For our processor of Poisson series, the terms $y_{n}$ are represented as pointers to list structures in which the trigonometric components are chained dynamically. The parity rules and the periodicity rules are all we need to know about the structures of the terms $y_{n}$ in order to construct the limit cycle. But, in Melvin's implementation, the Poisson series are represented by the static arrays of their numerical coefficients. Thus one needs to know that the term $y_{n}$ is a Fourier sum of the form

$$
y_{n}=\sum_{0 \leq m \leq} c_{n, m} \cos (2 m+1) \tau
$$

when $n$ is even, and of the form

$$
y_{n}=\sum_{1 \leq m \leq n} s_{n, m} \sin (2 m+1) \tau
$$

when $n$ is odd. Expressed in terms of the matrices $c_{n, m}$ and $s_{n, m}$, the algorithm of Lindstedt-Poincare gives rise to a large set of intricate algebraic formulas.

The tables on the next page present the development of the limit cycle to order 8 . We confirm that the corresponding coefficients produced by Melvin in floating point format are exact to 6 digits with rounding for the last digit. As the tables show, numerators and denominators in the coefficients of the cycle and of its frequency are rapidly increasing in absolute value. Our programs written in PL/1 for the IBM Optimizing Compiler (version 1, release 3.0) make use of the arithmetic hardware to operate on integers in decimal notation with at most 15 digits. Hence, the program encountered a fixed point overflow in the course of the operations at the 9-th order. A preliminary version in which the normalizing factorials were omitted in (1) and (2) raised the fixed overflow condition at order 5.

Another way of determining the limit cycle uses complex coordinates $(u, v)$. They are introduced by

$$
\begin{aligned}
& u=y-i \dot{y} \\
& v=y+i \dot{y}
\end{aligned}
$$

They transform van der Pol's equation into

$$
\dot{u}=i u-\frac{\epsilon}{8}(u-v)\left((u+v)^{2}-4\right)
$$

A conjugate complex equation holds for $v$. As the cycle in real form is a Fourier series with period $2 \pi / \omega$ we introduce a new independent variable by

$$
\xi=e^{i \omega t}
$$

The solution is then found as a formal power series of the form

$$
u=\sum_{n \geq 0} \epsilon^{n} u_{n}(\xi)
$$

where the $u_{n}(\xi)$ are odd polynomials in $\xi$. The frequency $\omega$ will be found at the same time in the form of the following series

$$
\omega=\sum_{n \geq 0} \omega_{n} \epsilon^{n}
$$

The new independent variable gives rise to the differential operator

$$
D=\xi \frac{d}{d \xi}=-\frac{1}{i \omega} \frac{d}{d t}
$$


TABLE I. Frequency.

\begin{tabular}{l|c}
\hline$n$ & $\omega_{n}$ \\
0 & 1 \\
2 & $\frac{1}{8}$ \\
4 & $\frac{17}{128}$ \\
6 & $\frac{175}{6144}$ \\
8 & $-\frac{4752293}{884736}$ \\
\hline
\end{tabular}

TABLE II. Terms of even order in the cycle.

\begin{tabular}{|c|c|c|c|c|c|}
\hline & $y_{0}$ & $y_{2}$ & $y_{4}$ & $y_{6}$ & $y_{8}$ \\
\hline \multirow{2}{*}{$\cos \tau$} & \multirow{18}{*}{2} & 1 & 23 & -258095 & \\
\hline & & 32 & 2048 & 1179648 & $c_{8}$ \\
\hline \multirow{2}{*}{$\cos 3 \tau$} & & 3 & 101 & 120305 & $\begin{array}{r}1958726609 \\
-\quad 1\end{array}$ \\
\hline & & & 512 & 196608 & 141557760 \\
\hline \multirow{2}{*}{$\cos 5 \tau$} & & 5 & 1865 & 1644175 & 3919930525 \\
\hline & & 48 & 4608 & 1769472 & 254803968 \\
\hline \multirow{2}{*}{$\cos 7 \tau$} & & & 1379 & -10923199 & 147344494843 \\
\hline & & & 4608 & 4423680 & 31850496000 \\
\hline \multirow{2}{*}{$\cos 9 \tau$} & & & 183 & -1769369 & 161113663733 \\
\hline & & & 2560 & 819200 & 5898240000 \\
\hline \multirow{2}{*}{$\cos 11 \tau$} & & & & -409871 & 1359229760383 \\
\hline & & & & 460800 & 46448640000 \\
\hline \multirow{2}{*}{$\cos 13 \tau$} & & & & 715247 & 2076538440769 \\
\hline & & & & 5160960 & 130056192000 \\
\hline \multirow{2}{*}{$\cos 15 \tau$} & & & & & 526426361 \\
\hline & & & & & 115605504 \\
\hline \multirow{2}{*}{$\cos 17 \tau$} & & & & & 392636471 \\
\hline & & & & & 743178240 \\
\hline
\end{tabular}

TABLE III. Terms of odd order in the cycle.

\begin{tabular}{l|c|c|c|c}
\hline \hline & $v_{1}$ & $y_{3}$ & $r_{5}$ & $r_{7}$ \\
\hline $\sin 3 \tau$ & $-\frac{1}{4}$ & $\frac{45}{256}$ & $-\frac{3895}{49152}$ & $\frac{31766119}{9437184}$ \\
$\sin 5 \tau$ & & $\frac{85}{384}$ & $-\frac{40475}{55296}$ & $-\frac{43837325}{42467328}$ \\
$\sin 7 \tau$ & $\frac{7}{96}$ & $-\frac{99967}{110592}$ & $\frac{2911646591}{530841600}$ \\
$\sin 9 \tau$ & & & $-\frac{9791}{20480}$ & $\frac{820810921}{98304000}$ \\
$\sin 11 \tau$ & & & $-\frac{5533}{61440}$ & $\frac{1657839733}{294912000}$ \\
$\sin 13 \tau$ & & & & $\frac{21731177}{11468800}$ \\
$\sin 15 \tau$ & & & & $\frac{693485}{2752512}$ \\
\hline
\end{tabular}


It allows us to rewrite eq (4) as

$$
D u=u+\frac{i \epsilon}{8}(u-v)\left((u+v)^{2}-4\right)-(\omega-1) D u
$$

From this equation the following parity rules can be read off immediately:

a) For $n$ even $u_{n}$ has purely real coefficients

b) For $n$ odd $u_{n}$ has purely imaginary coefficients and $\omega_{n}=0$

Starting with

$$
u_{0}=2 \xi, \quad u_{1}=i\left(1 / 2 \xi^{3}+1 / 4 \xi^{-3}\right) \quad \text { and } \quad \omega_{0}=1
$$

the coefficients $\omega_{n}$ and the polynomials can be determined recursively by the following method:

For even $n$ the terms of order $\epsilon^{n}$ in eq (5) give rise to the differential equation

$$
D u_{n}=u_{n}+F_{n}(\xi)-2 \omega_{n} \xi
$$

$F_{n}(\xi)$ is a known polynomial since it depends only on $u_{k}(\xi)$ and $\omega_{k}$ with $k<n$.

It will contain a linear term with a real coefficient $a$ so that $F_{n}(\xi)=a+F_{n}^{*}(\xi)$. Since $\xi$ is a solution to the homogeneous equation this is a critical term and we have to set $\omega_{n}=a / 2$. The general solution to the remaining equation

$$
D u_{n}=u_{n}+F_{n}^{*}(\xi)
$$

is then

$$
u_{n}=\alpha \xi+u_{n}^{*}(\xi)
$$

where $u_{n}^{*}(\xi)$ is a polynomial in $\xi$. The parameter $\alpha$ will be determined at the next order. The differential equation for terms of odd order $n+1$ reads

$$
D u_{n+1}=u_{n+1}+F_{n+1}(\xi)+i \alpha\left(\xi+\frac{3}{2} \xi^{3}-\xi^{-1}-\frac{3}{2} \xi^{-3}\right) .
$$

$F_{n+1}(\xi)$ denotes again a known polynomial which will contain a linear factor of the form $i b \xi$. To avoid secular terms we require $\alpha=-b$. The remaining equation can then be integrated to give the polynomial $u_{n+1}$. The solution to the homogeneous solution is set to zero this time out of convenience and to obtain the phase shifting solutions of [1].

The second author used his algebraic processor POLYPAK to implement the above algorithm by computer and to check the results in [1]. POLYPAK is a package of PL/I computer programs for the manipulation of real or complex powerseries in several variables. It was derived from MAO. All computations were performed on the Amdahl 470/V6 of the University of Cincinnati which runs under the IBM operating system VS2 release 1.7 .

For the above problem our series had two variables $\epsilon$ and $\xi$ and the coefficients were stored as complex double precision floating point numbers. We determined the solution $u$ and the series $\omega$ through terms of order $\epsilon^{45}$ and confirmed the results given in [1].

Our computations took 57 seconds. This compares with 130 seconds for a FORTRAN program which Melvin used to implement his formulas and which he ran on a CYBER 175. A comparison of the two times may not mean very much as we compare different machines and different programs. Nevertheless, we believe that it indicates that the use of POLYPAK did not generate any unreasonable overhead. The use of an algebraic processor is already justified by the ease with which the above algorithm can be coded.

\section{References}

[1] Melvin, P. The Phase-Shifting Limit Cycles of the Van der Pol's Equation, J. Res. Nat. Bur. Stand. (U.S.) 83, No. 6, 593-602 (Nov.-Dec. 1978). 